Check for updates

Cite this: RSC Adv., 2019, 9, 278

Received 1st August 2018

Accepted 10th December 2018

DOI: $10.1039 / \mathrm{c} 8 \mathrm{ra06493h}$

rsc.li/rsc-advances

\section{Preparation of super-hydrophilic polyphenylsulfone nanofiber membranes for water treatment $\uparrow$}

\author{
Yan Wang, ${ }^{\text {ab }}$ Radoslaw Pawel Górecki, ${ }^{\text {cd }}$ Eugen Stamate, ${ }^{e}$ Kion Norrman, \\ David Aili, (D) Min Zuo, (D) Weihong Guo, (D) *a Claus Hélix-Nielsen*c \\ and Wenjing Zhang $(\mathbb{D}$ *e
}

Electrospun nanofiber membrane-supported thin film composite (TFC) membranes exhibit great potential in water purification. In this work, electrospun polyphenylsulfone (PPSU) nanofiber membranes were prepared and modified by heat and plasma treatments. The resulting membranes were used as support layers for biomimetic TFC-based forward osmosis membranes. Thermal treatment transformed a loose non-woven nanofiber structure into a robust interconnected 3-dimensional PPSU network displaying a 930\% increase in elastic modulus, $853 \%$ increase in maximum stress, and two-fold increase in breaking strain. Superior hydrophilicity of PPSU nanofiber membranes was achieved by low-pressure plasma treatment, changing the contact angle from $137^{\circ}$ to $0^{\circ}$. The fabricated exemplary TFC-based forward osmosis membrane showed an osmotic water flux $J_{w}>14 \mathrm{~L} \mathrm{~m}^{-2} \mathrm{~h}^{-1}$ with a very low reserve salt flux $J_{s}$ $\left(J_{s} / J_{w}=0.08 \mathrm{~g} \mathrm{~L}^{-1}\right)$ demonstrating the potential for making high quality membranes for water treatment using PPSU-based support layers for TFC membranes.

\section{Introduction}

Electrospinning offers absolutely unique possibilities of structuring complex networks at the nanoscale, using a rich variety of materials (e.g. polymer, ceramics and composite) and with the ability to control composition, morphology and even secondary structure. ${ }^{1-3}$ Due to the specialized properties, relatively facile fabrication and flexible modification, electrospun nanofibers have shown huge potential in various applications, such as fuel cells, ${ }^{4-8}$ sensors, ${ }^{9}$ catalysts, ${ }^{10}$ drug delivery, ${ }^{11}$ nanofiber reinforced composites, ${ }^{12}$ and water purification. ${ }^{13-16}$

${ }^{a}$ Polymer Processing Laboratory, Key Laboratory for Preparation and Application of Ultrafine Materials of Ministry of Education, School of Material Science and Engineering, East China University of Science and Technology, Shanghai, People's Republic of China

${ }^{b}$ Department of Chemical Engineering, Huaihai Institute of Technology, Lianyungang, People's Republic of China

'Department of Environmental Engineering, Technical University of Denmark, Miljøvej 113, DK-2800 Kongens Lyngby, Denmark

${ }^{d}$ Aquaporin A/S, Nymøllevej 78, DK-2800 Kongens Lyngby, Denmark

${ }^{e}$ Department of Energy Conversion and Storage, Technical University of Denmark, Elektrovej 375, DK-2800 Kongens Lyngby, Denmark.E-mail: wenz@dtu.dk

${ }^{f} M O E$ Key Laboratory of Macromolecular Synthesis and Functionalization, Department of Polymer Science and Engineering, Zhejiang University, Hangzhou, People's Republic of China

$\dagger$ Electronic supplementary information (ESI) available. See DOI: 10.1039/c8ra06493h
In recent years, electrospun nanofiber membranes (ENMs) have drawn increasing attention as supporting layers used in thin film composite (TFC) membrane systems for water purification. In this connection, the TFC membranes combine high rejection ratio and permeability with mechanical strength and low cost. They have thus been widely used in water filtration, including ultrafiltration (UF), nanofiltration (NF) and reverse osmosis (RO). ${ }^{13,15}$ The membrane system usually consists of a non-woven support substrate, a porous polymer layer offering either filtration or support function and a selective layer with nanoscale pore size. Compared to conventional porous polymer membranes manufactured by phase inversion method, ENMs have relatively high porosity and interconnected porous structure. These features make them a good substitute for the porous polymer layer in TFC membranes. The group of Chu and Hsiao $^{17-20}$ has reported a series of thin film nanofiber composite (TFNC) UF and NF membranes having a hydrophilic separation layer, an electrospun nanofibrous scaffold as middle layer and a nonwoven support layer. These TFNC membranes exhibited significantly higher flux rate compared to commercial UF membranes while retaining a similar rejection rate in the separation of oil emulsions and water.

Beyond the classical UF, NF and RO processes, ENMs may also be of particular interest to the emerging forward osmosis (FO) membrane technology. In FO, the driving force for water flux $J_{\mathrm{w}}$ is determined by the effective osmotic pressure differential across the membrane separating a so-called draw solution (DS) from a feed solution (FS). As long as the osmotic 
pressure of the DS exceeds that of the FS, $J_{\mathrm{w}}>0$ and the DS will be gradually diluted while the FS will be concentrated. The absence of a hydraulic pressure differential used in UF, NF, and RO brings certain benefits to FO such as lower membrane fouling propensity and opens for applications in food and pharmaceutical processes. ${ }^{21,22}$

However, since an ideal FO membrane has high water permeability (from FS to DS) with concomitant low reverse solute (from the concentrated DS into the FS), it can be seen as a particularly demanding membrane design challenge. The appearance of unstirred layers inside the FO membrane material, referred to as internal concentration polarization (ICP), will tend to reduce the effective osmotic driving force across the separation (or active) region in the material. Indeed FO membranes can be made using conventional TFC membranes with phase inversion support layers. But these membranes suffer from severe ICP problems due to the relatively low porosity (and high tortuosity), resulting in poor water flux performance in FO mode.

Thus a thin and highly porous structure is desirable - but this contrasts the need for good overall mechanical strength and chemical stability of the membrane, creating an ICP bottleneck. $^{22}$ ENMs with high porosity and interconnected porous structure could provide a good solution for eliminating the ICP bottleneck. ${ }^{23}$ The McCutcheon group has reported TFNC FO membranes made of polysulfone, ${ }^{24}$ polyethersulfone, ${ }^{24}$ polyacrylonitrile/cellulose acetate blend ${ }^{25}$ and nylon-6,6. ${ }^{26}$ Compared to commercial FO membranes, the TFNC FO membranes showed enhanced water flux. Also Wang's group has achieved high-performance TFNC FO membranes based on polyvinylidene fluoride (PVDF), ${ }^{27}$ polyetherimide reinforced by carbon nanotubes ${ }^{\mathbf{2 8}}$ and polyetherimide embedded with silica nanoparticles ${ }^{29}$ as electrospun nanofiber support.

In addition to high porosity and an interconnected porous structure, hydrophilicity of the support layer was found to play a crucial role in water flux across the FO membrane. ${ }^{30}$ Although water flux was enhanced in TFNC FO membranes with hydrophilic ENMs as supports, a decrease in strength, due to fibre swelling in water, was also noted. ${ }^{26}$ One way to mitigate this is to modify the surface of hydrophobic fibres, ${ }^{31}$ where the chosen hydrophobic polymer offers good mechanical strength and chemical stability, and where the surface is modified to be hydrophilic. Huang et $a .^{31}$ reported chemical surface modification of electrospun PVDF mats involving interfacial polymerization to form nylon-6,6 onto the nanofiber surface. TFNC FO membranes with modified PVDF nanofibrous supports exhibited good performance (in terms of $J_{\mathrm{w}}$ and $J_{\mathrm{s}}$ ). However, the surface modified membranes in this study were only moderately hydrophilic (contact angle $48^{\circ}$ ) and had an elastic modulus of $26 \mathrm{MPa}$.

In the present study, polyphenylsulfone (PPSU) ENMs were fabricated and used as support layers in TFNC FO membranes after post treatments. Heat treatments were applied to enhance the integrity and mechanical strength, and plasma treatments to increase the surface wettability of the ENMs. The effects of post treatments on PPSU ENMs properties were investigated, and X-ray photoelectron spectroscopy (XPS) was employed to monitor ageing effects in PPSU ENMs. PPSU-based FO membrane's functionality was tested by depositing a biomimetic polyamide active layer with incorporated aquaporin onto the surface of heat-treated PPSU ENM by interfacial polymerization. The separation properties of the resulting TFNC FO membrane were characterized in FO mode.

\section{Materials and methods}

\subsection{Materials}

The PPSU polymer Radel ${ }^{\circledR}$ R-5500NT (density $=1.29 \mathrm{~g} \mathrm{~cm}^{-3}$, glass transition temperature $=220^{\circ} \mathrm{C}$ ) was supplied by Solvay Advanced Polymer LLC. $N$-methyl-2-pyrrolidone (NMP) and acetone were supplied from Sigma-Aldrich. 1,3-Phenylenediamine (MPD) (98.0\% for synthesis) and 1,3,5-benzenetricarbonyl chloride (TMC) (97.0\% for synthesis) were obtained from Merck KGaA. The Aquaporin Inside ${ }^{\mathrm{TM}}$ nanostructures solution was prepared accordingly to a patented technology. ${ }^{32}$ Isopar $^{\mathrm{TM}} \mathrm{E}$ was obtained from Brenntag Nordic $\mathrm{A} / \mathrm{S}$ and filtered prior to use on glass filter with porosity of 1-100 m. Calcein was supplied by Sigma-Aldrich, and $\mathrm{NaCl}$ was supplied by AkzoNobel. All materials were used as received.

\subsection{Electrospinning PPSU}

PPSU was dissolved in a binary solvent system of NMP/acetone with a weight ratio of $8: 2$. The mixture was stirred at room temperature for $24 \mathrm{~h}$, to obtain homogenous solutions with a solid content of $30 \mathrm{wt} \%$.

PPSU ENMs were prepared by an electrospinning apparatus purchased from Linari Nanotech. The polymer solutions were transferred into a $10 \mathrm{~mL}$ plastic syringe, and fed through a metal needle with an inner diameter of $0.8 \mathrm{~mm}$ at a constant flow rate of $0.2 \mathrm{~mL} \mathrm{~h}^{-1}$. The positive electrode of the high voltage power supply was connected to the metal needle. The grounded electrode was connected to a stainless steel drum, wrapped with silicon coated kraft paper, rotating at $100 \mathrm{rpm}$. During the electrospinning, the applied voltage and the collecting distance were $15 \mathrm{kV}$ and $12 \mathrm{~cm}$, respectively. The relative humidity and temperature were kept at $19-22 \%$ and $29-30{ }^{\circ} \mathrm{C}$, respectively.

\subsection{Post treatments of the PPSU ENMs}

The heat treatment of the as-spun PPSU ENMs was conducted in oven at $240{ }^{\circ} \mathrm{C}$ for $1 \mathrm{~h}$. The sides of the membranes were fixed during the heat treatment in order to prevent the shrinkage.

The ENMs were treated in a low-pressure plasma produced in a cubical vacuum chamber of $40 \times 40 \times 40 \mathrm{~cm}^{3}$, equipped with 12 electron cyclotron resonance (ECR) plasma cells ${ }^{33}$ powered by microwaves at $2.45 \mathrm{GHz}$. A more detailed description is presented elsewhere. ${ }^{34}$ The magnetic filter used to reduce the electron temperature was removed for this set of experiments. The discharge power was set at $1000 \mathrm{~W}$ and the oxygen gas pressure at $0.67 \mathrm{~Pa}$. It corresponded to $9.7 \times 10^{15} \mathrm{~m}^{-3}$ for plasma density, $0.9 \mathrm{eV}$ for electron temperature and $8.3 \mathrm{~V}$ for plasma potential, as measured by a cylindrical Langmuir probe. ${ }^{35}$ The $\mathrm{O}_{2}$ dissociation rate was evaluated at $30 \%$ using 
a HIDEN mass spectrometer. The $5 \times 10 \mathrm{~cm}^{2}$ membranes were placed $15 \mathrm{~cm}$ below the ECR plasma cells, at the centre of the vacuum chamber, and have been exposed to plasma discharge for $2 \mathrm{~min}$. on each side. Due to moderate plasma density and low-pressure operation, the membranes were exposed to a high concentration of atomic oxygen while the heating was kept below $70{ }^{\circ} \mathrm{C}$.

\subsection{Characterization of PPSU ENMs}

Surface morphology of both modified and unmodified PPSU ENMs was investigated by a Zeiss Supra 55VP scanning electron microscope (SEM). The samples were coated with gold before imaging to obtain better contrast and to avoid charge accumulation. The fibre diameter distributions and average fibre diameters were determined using ImageJ software. More than 100 fibres were analysed to get the average fibre diameter and a histogram of the diameter distribution.

The porosity of both modified and unmodified PPSU ENMs was investigated by a POREMASTER mercury porosimetry analyser from Quantachrome Instruments.

To investigate the wettability of modified and unmodified PPSU ENMs, water contact angles of the membranes were measured by a DSA25 drop shape analyser from Krüss.

Tensile tests were performed by applying a universal testing machine at room temperature with a crosshead speed of 10 $\mathrm{mm} \mathrm{min}^{-1}$. All the samples were conditioned in air for more than $24 \mathrm{~h}$ before test. The specimens, $10.0 \mathrm{~mm}$ wide and 42.0$48.0 \mathrm{~mm}$ long, were prepared by die-cutting using a hydraulic press. Each sample was measured at least ten times.

The thickness of the membranes was measured by CT100 profilemeter from Cyber Technologies. 3D measurements were employed on the membranes, and the average values of was taken as the final thickness. The thicknesses of the as-spun and heat-treated PPSU ENMs are $380 \mu \mathrm{m}$ and $310 \mu \mathrm{m}$, respectively.

To monitor ageing effects in PPSU ENMs, XPS analyses were performed on a ESCALAB XI ${ }^{+}$X-ray photoelectron spectrometer microprobe (Thermo Fisher Scientific, East Grinstead, U.K.) using a monochromatic Al-K $\alpha$ X-ray source with a $650 \mu \mathrm{m}$ spot size and a take-off angle of $90^{\circ}$ from the surface plane. Atomic concentrations were determined from survey spectra (0$1350 \mathrm{eV}, 100 \mathrm{eV}$ detector pass energy, $1 \mathrm{eV}$ step size, $50 \mathrm{~ms}$ dwell time, 3 scans) and were calculated by determining the relevant integral peak intensities using a Smart type background. Five different surface locations were analysed on each sample surface and average values were calculated.

\subsection{Formation of polyamide active layer}

The selective polyamide active layer of the thin film composite membrane was prepared by interfacial polymerization. Firstly, the PPSU ENM was soaked in an aqueous solution of $2.5 \mathrm{wt} \%$ MPD containing $0.5 \mathrm{wt} \%$ of Aquaporin Inside ${ }^{\mathrm{TM}}$ nanostructures for 30 seconds. Subsequently, the membrane was placed on the glass plate and the excess of the aqueous phase was removed by an air-knife set up to 1 bar. The membrane was immobilized and its sides were sealed by sticking it to the glass plate with tape. Such immobilized membrane was immersed in a $0.21 \mathrm{wt} \%$
TMC organic solution of Isopar ${ }^{\mathrm{TM}} \mathrm{E}$ for 30 seconds. The membrane was then dried by the air-knife set up to 1 bar for 15 seconds, detached from the glass plate and stored in reverseosmosis purified water overnight prior to testing.

\subsection{Separation properties of the TFNC FO membrane}

Separation properties of the resulting TFNC FO membrane were characterized in a standard FO setup as shown in Fig. 1. Two peristaltic pumps were applied to circulate the feed (DI water with forward rejection tracer e.g. $5 \mu \mathrm{M}$ calcein) and draw solution (1 $\mathrm{M} \mathrm{NaCl}$ aqueous solution) with a counter-current crossflow configuration. The membrane was placed in the chamber with an active area of approximately $33 \mathrm{~cm}^{2}$. The weight loss from the feed was monitored by a balance, and the concentration change from the draw by a conductivity probe. The running time was $200 \mathrm{~min}$.

The osmotic water flux, $J_{\mathrm{w}}\left(\mathrm{L} \mathrm{m}^{-2} \mathrm{~h}^{-1}\right)$ was calculated according to eqn (1), where $\Delta w(\mathrm{~kg})$ is the weight change of the feed over $\Delta t(\mathrm{~h}), A_{\mathrm{m}}\left(\mathrm{m}^{2}\right)$ is the active area of the membrane, and $\rho\left(\mathrm{kg} \mathrm{L}^{-1}\right)$ is the density of water.

$$
J_{\mathrm{w}}=\frac{\Delta w}{A_{\mathrm{m}} \Delta t \rho}
$$

The salt reverse flux, $J_{\mathrm{s}}\left(\mathrm{g} \mathrm{m}^{-2} \mathrm{~h}^{-1}\right)$ was derived from the eqn (2), where $C_{0}\left(\mathrm{~g} \mathrm{~L}^{-1}\right)$ and $V_{0}(\mathrm{~L})$ are the salt concentration and feed volume at the starting point, $C_{\mathrm{t}}$ and $V_{\mathrm{t}}$ are the final salt concentration and feed volume over $\Delta t$.

$$
J_{\mathrm{s}}=\frac{C_{\mathrm{t}} V_{\mathrm{t}}-C_{0} V_{0}}{A_{\mathrm{m}} \Delta t}
$$

\section{Results and discussion}

\subsection{Effects of the heat treatment on ENM morphology and fibre diameter distribution}

SEM images of PPSU ENMs surfaces before and after heat treatment are shown in Fig. 2 and 3, respectively. As shown in Fig. 2, electrospinning of the solution containing $30 \mathrm{wt} \%$ PPSU in the binary solvent system of NMP/acetone at $8: 2$ weight ratio led to thin fibres free of beads. The fibres did not adhere with each other at the intersections and formed a loose non-woven structure, as they were dry when deposited on the collector, with solvents evaporated during the electrospinning process.

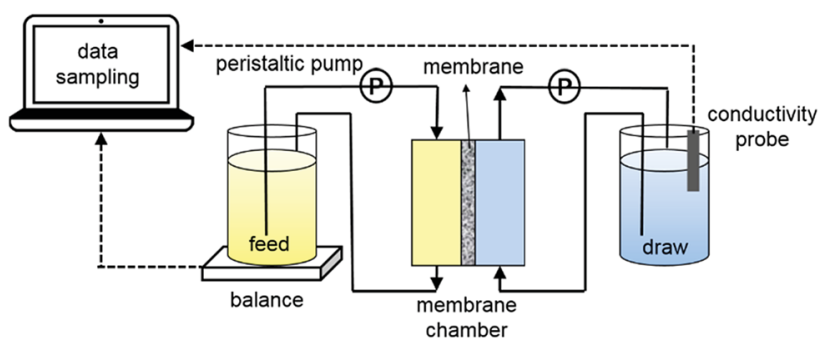

Fig. 1 Schematic diagram of forward osmosis setup. 

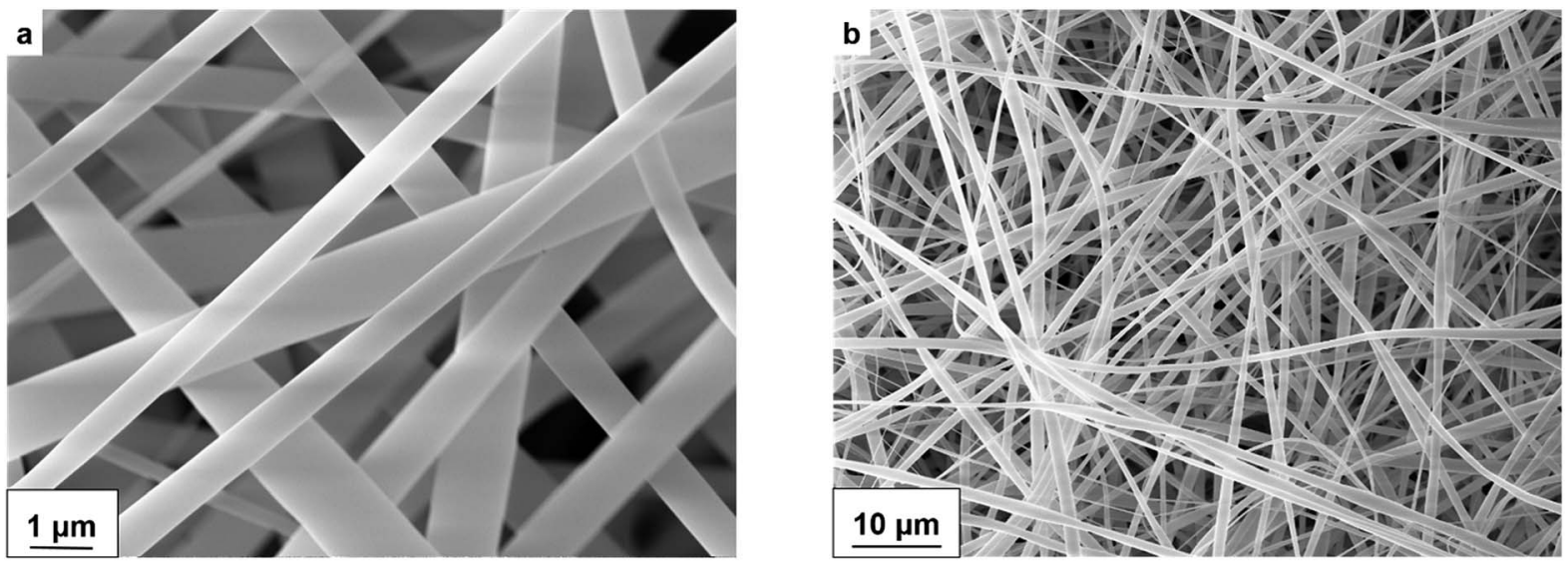

Fig. 2 SEM images of as-spun PPSU ENMs surfaces: (a) $35000 \times$, (b) $5000 \times$

The post thermal treatment is often used to improve the electrospun membrane properties such as pore size distribution, mechanical or thermal properties. ${ }^{16}$ In order to improve the integrity and mechanical strength of PPSU ENM, heat treatment was applied at $240{ }^{\circ} \mathrm{C}$ for $1 \mathrm{~h}$ after electrospinning. As can be seen from Fig. 3, welding joints were formed at the intersections of the fibres during the heat treatment, and fusion along the fibre length could also be observed. The loose nonwoven nanofiber structure of the as-spun membranes has been transferred into interconnected 3-dimensional porous network by the heat treatment.

Fibre diameter distributions and average fibre diameters of as-spun and heat-treated PPSU ENMs are summarized in Fig. 4. As reported in ref. 36, the average fibre diameter of the heattreated PPSU ENM was lower than that of the untreated one, due to the rearrangement of polymer chains caused by external force during the heat treatment. However, in our work, the average fibre diameter of the heat-treated ENM was $23 \%$ higher than that of the as-spun one. During electrospinning, the polymer solution jet was stretched under electrostatic force. Therefore, internal stress remained in the generated fibres. During heat treatment, the PPSU fibres were heated above its glass transition temperature, at which the segmental motion of the polymer chain was activated. The motion of segments in polymer chain tends to relax the internal stress, causing the lengthwise shrinkage and radial expansion of the fibres. Moreover, the sides of the membrane were fixed to prevent irregular shrinkage. Therefore, some of the fibres were broken by the tension, as shown in Fig. 2(b). Also, most of the very thin fibres (with the diameter less than $400 \mathrm{~nm}$ ) disappeared in the heat-treated membrane, as shown in Fig. 4, because they were more likely to break and fuse with adjacent ones. Therefore the average fibre diameter increased after heat treatment as the result of both segmental motion of polymer chains and break of fibres (especially thinner ones).

\subsection{Effects of heat treatment on ENM mechanical properties}

Mechanical properties of the as-spun and heat-treated PPSU ENMs were investigated by tensile test. Fig. 5 shows the representative stress-strain curves of PPSU ENMs before and after the heat treatment. Compared to the as-spun membrane, the heat-treated one exhibited a much steeper increase of the stress in the initial part of the curve, which indicated an enhancement in mechanical modulus. Also, a much higher stress peak and

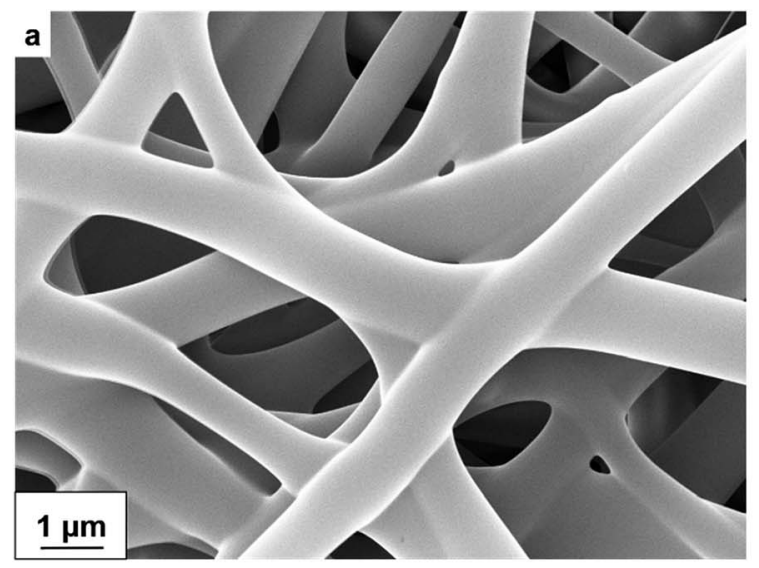

Fig. 3 SEM images of heat-treated PPSU ENM surfaces: (a) $35000 \times$, (b) 5000x, the circles indicate the break of fibres.

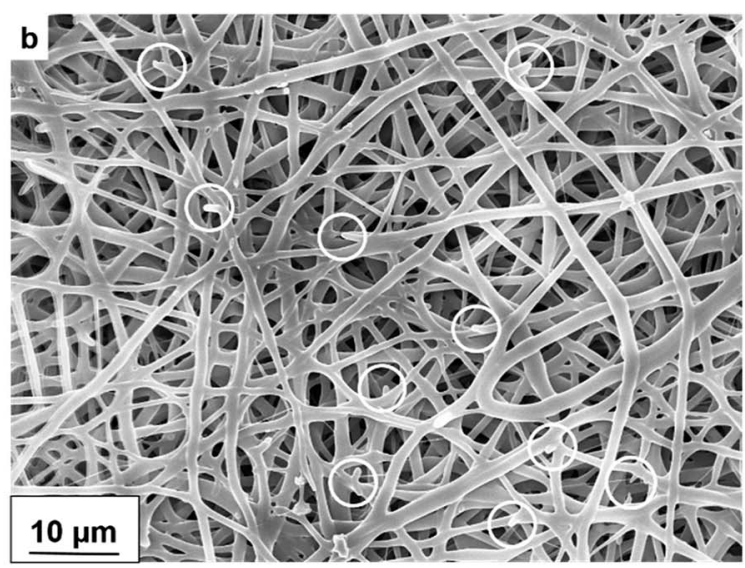



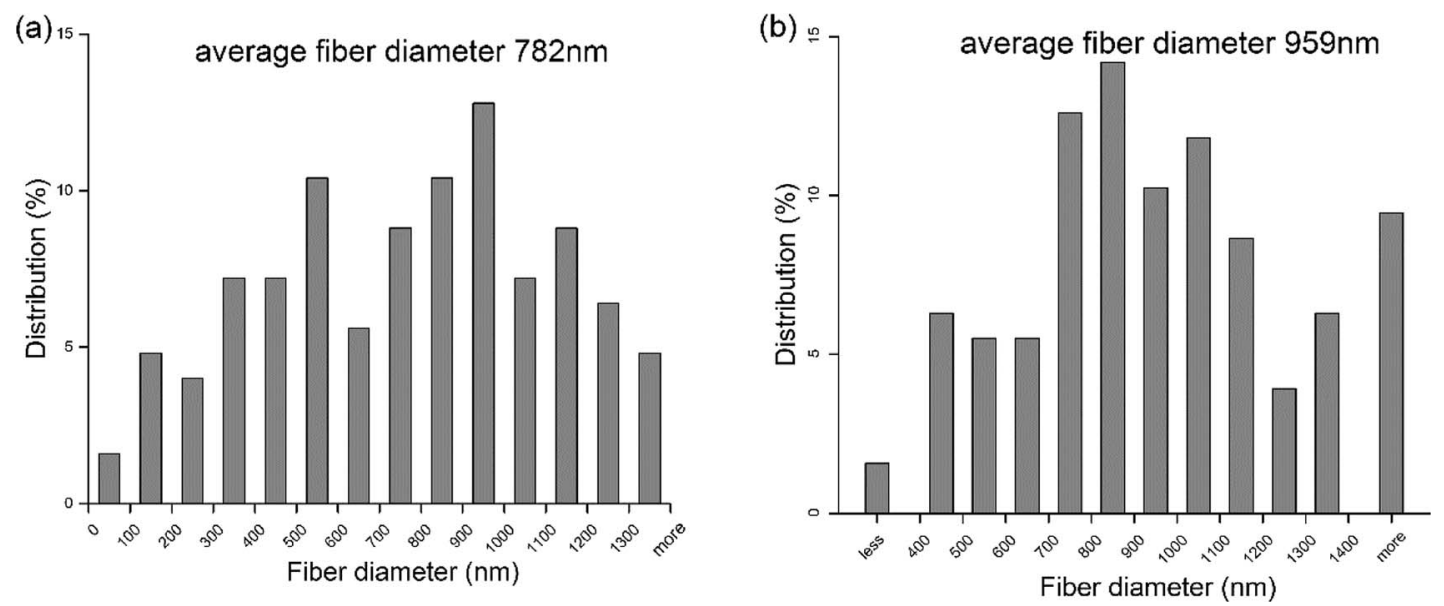

Fig. 4 Fibre diameter distribution of as-spun PPSU ENMs (a) and heat-treated PPSU ENMs (b).

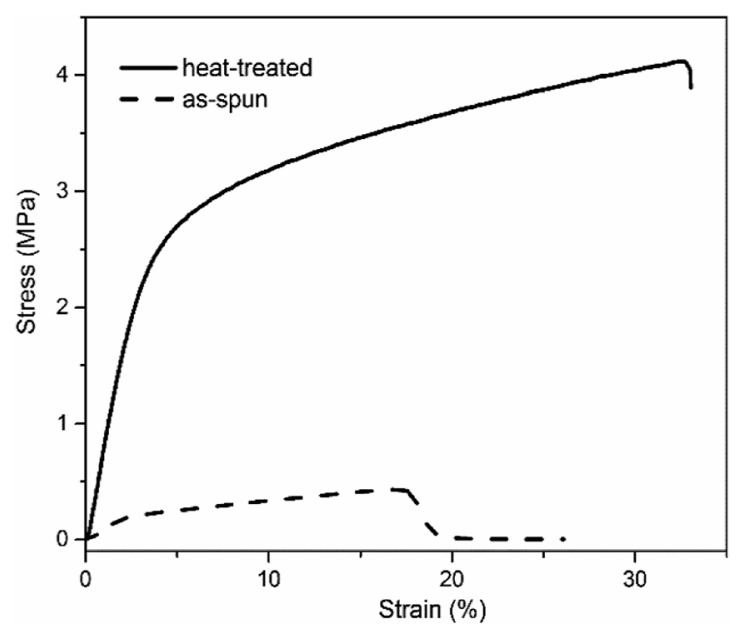

Fig. 5 Representative stress-strain curves of the as-spun and heattreated PPSU ENMs.

larger elongation at break were observed for the heat treated membranes, showing that the heat treatment has enhanced both the stiffness and ductility of the membranes.

Tensile properties of the as-spun and heat-treated PPSU ENMs are summarized as the average values and corresponding standard deviations for 10-11 samples, and listed in Table 1. All the three tensile properties of the heat-treated membranes were increased significantly compared to those of as-spun membranes. The elastic modulus of the heat-treated membrane was found to be $930 \%$ higher than that of the asspun membrane, and the breaking stress $853 \%$ higher. The breaking strain roughly doubled after heat treatment. The asspun PPSU membrane had a loose structure with poor cohesiveness because the fibres did not adhere with each other, as shown in Fig. 2. During the heating process, polymer fibres were fused together at the intersections to form a more compact and integrate structure. Moreover, the segmental motions of the polymer chains during the heat treatment efficiently removed the internal stress formed in the spinning process, resulting in the enhancement of structural stability. Also, the segmental motions could rearrange the polymer chains along the direction of the external force applied by fixing the sides of the membranes and increased the orientation degree of the amorphous polymer. All of these facilitated the enhancement of structural stability and the improvement of mechanical properties. Similar performances have been reported previously. ${ }^{36-39}$

\subsection{Effects of heat treatment on ENM porosities}

Pore size distributions of the as-spun and heat-treated PPSU ENMs are illustrated in Fig. 6. Both membranes showed relatively narrow pore size distribution, while the heat treatment led to an increase in the pore size. During heat treatment, as shown in Fig. 3, those very thin fibres broke and fused with other fibres nearby, which was the cause of the increase in the pore size. However, the porosity of the membrane reduced after heat treatment, from $96 \%$ to $68 \%$. The high porosity of the as-spun membrane could be the result of a fully interconnected open pore structure. Then, during heat treatment, fibres were fused together, resulting in a more compacted membrane structure with less space among nanofibers.

Table 1 Tensile properties of the as-spun and heat-treated PPSU ENMs

\begin{tabular}{lccc}
\hline Samples & Elastic modulus $(\mathrm{MPa})$ & Stress at break (MPa) & Strain at break (\%) \\
\hline As-spun & $8.7 \pm 2.0$ & $0.43 \pm 0.05$ & $17.7 \pm 0.9$ \\
Heat-treated & $89.6 \pm 3.7$ & $4.10 \pm 0.2$ & $34.4 \pm 4.5$
\end{tabular}




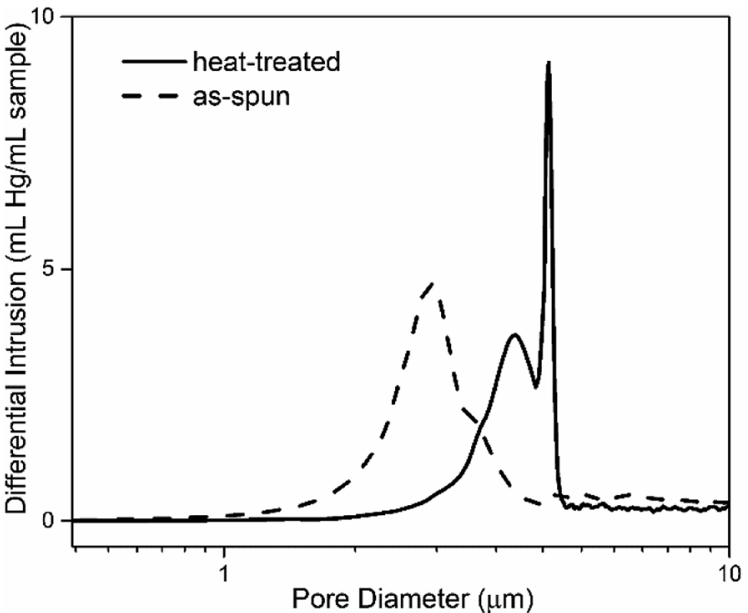

Fig. 6 Pore size distributions of the as-spun and heat-treated PPSU ENMs.

\subsection{Effects of plasma treatment on ENM wettability}

Plasma treatment has been utilized as a promising method for surface modification of many materials, such as polymers, ${ }^{40-43}$ carbon materials, ${ }^{44-46}$ magnetic materials ${ }^{47}$ and other naturalbased materials. ${ }^{48}$ The results of the plasma treatments can be tailored by changing the gas phase. ${ }^{44}$ Oxygen plasma treatment was found to be an effective tool to modify surface wettability. ${ }^{40,42,43}$ As-spun PPSU ENMs were hydrophobic, which made it difficult to coat the biomimetic active layer (incorporated with aquaporins) onto the membranes, which could hinder the water flux in the FO process. ${ }^{30}$ Therefore, plasma treatments were applied to improve the wettability of the membranes. Water contact angles of the PPSU ENMs before and after plasma treatment were measured in order to investigate the surface modification. Fig. 7 shows the difference between the contact angles of PPSU ENMs before and after post treatments. Both the as-spun membrane and the heat-treated membrane have a hydrophobic nature, and the contact angle of the heat-treated membrane slightly decreased compared to that of as-spun membrane. This could be the result of the smoother and more compacted surfaces of the heat-treated samples. For the plasma-treated membranes, as shown in Fig. 7(c), static contact angle measurements were unable to

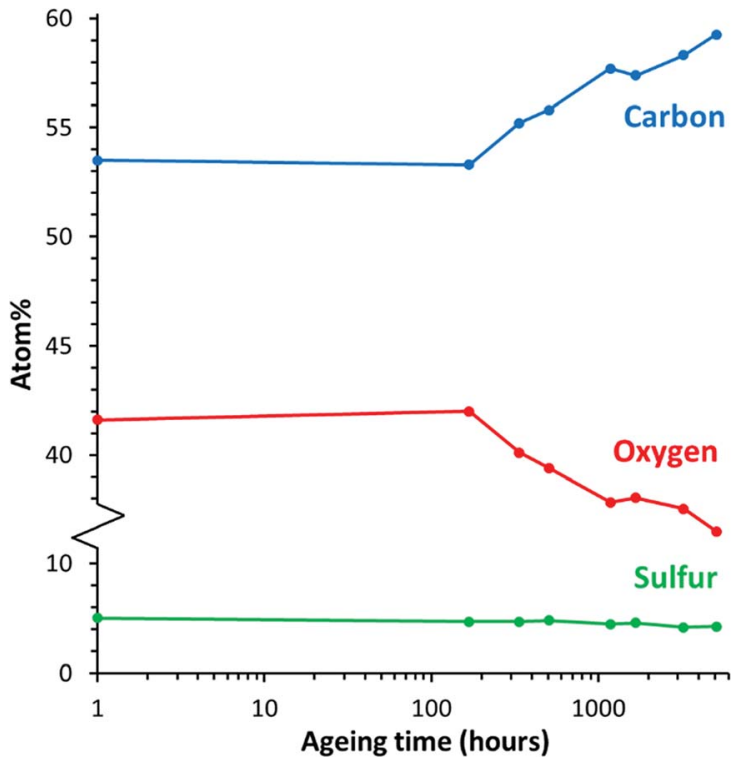

Fig. 8 A plot of the $\mathrm{C} / \mathrm{O} / \mathrm{S}$ composition for the PPSU ENM that was plasma treated as a function of time in darkness at room temperature in ambient air.

perform because the water drop disappeared into the membrane immediately, indicating that the membrane surface has turned to be super hydrophilic after plasma treatment.

The plasma treated PPSU ENMs were studied using XPS. The carbon/oxygen/sulphur compositions (hereafter termed C/ $\mathrm{O} / \mathrm{S}$ ) were monitored as a function of time. In between analyses, the two samples were stored in ambient air in darkness at room temperature. The result is shown in Fig. 8. The plasma treatment oxidizes the PPSU polymer, i.e. degrades the molecular structure to some extent, and introduces carbon functionalities that, depending on the functionality, have varying degree of hydrophilicity. The oxygen content is therefore expected to increase during plasma treatment. As a result of increasing the oxygen content, the relative carbon content is expected to decrease. This is consistent with the observations (not shown). Detailed investigation of plasma-induced chemical changes of the PPSU ENMs are presented in our work elsewhere. ${ }^{49}$ When monitoring the effect of time on the composition, an ageing effect is observed. The element
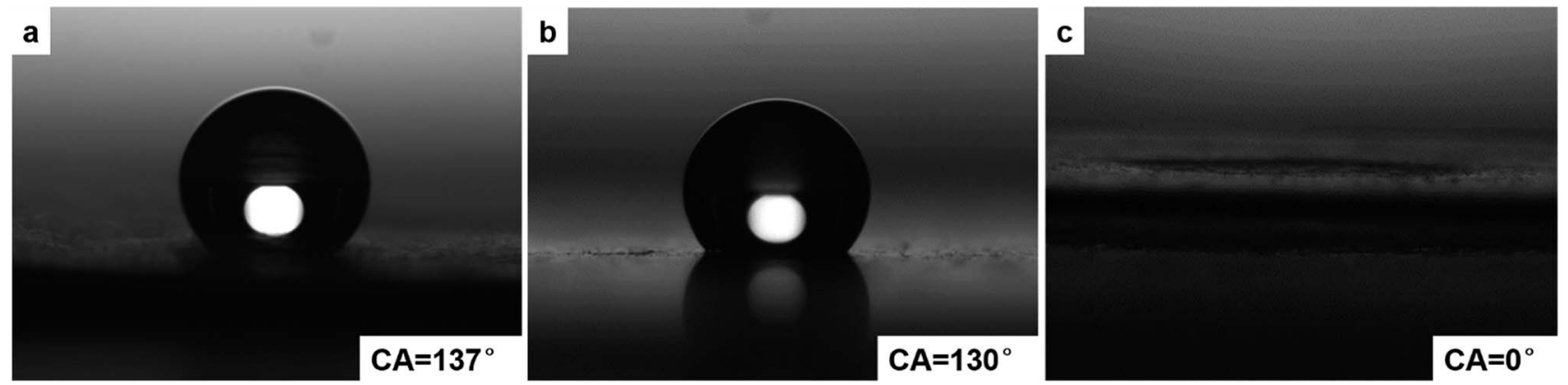

Fig. 7 Contact angles of water droplets on the PPSU ENMs. (a) As-spun membrane. (b) Heat-treated membrane. (c) Heat-treated and plasmatreated membrane. 
Table 2 Separation properties of Aquaporin-coated TFNC FO membrane with PPSU ENM and commercial membrane as substrate

\begin{tabular}{|c|c|c|c|c|}
\hline Substrate & $J_{\mathrm{w}}\left(\mathrm{L} \mathrm{m}^{-2} \mathrm{~h}^{-1}\right)$ & $J_{\mathrm{s}}\left(\mathrm{g} \mathrm{m}^{-2} \mathrm{~h}^{-1}\right)$ & $R_{\mathrm{ca}}(\%)$ & $J_{\mathrm{s}} / J_{\mathrm{w}}\left(\mathrm{g} \mathrm{L}^{-1}\right)$ \\
\hline PPSU ENM & 14.25 & 1.15 & 99.8 & 0.08 \\
\hline $\begin{array}{l}\text { Commercial } \\
\text { membrane }^{a}\end{array}$ & $>10$ & $<3$ & $>99.0$ & $<0.3$ \\
\hline
\end{tabular}

composition remains unchanged for at least $168 \mathrm{~h}$, thereafter the measured oxygen content starts to decrease, and the relative carbon content is observed to increase. The sulphur content is only slightly affected. The ageing effect is in progress even after $5088 \mathrm{~h}$, where the experiment was terminated.

A possible explanation to the observed development in the element composition could be that oxidized PPSU (or oxidized PPSU fragments) diffusing into the bulk and at the same time non-oxidated PPSU diffusing out from the bulk to the surface. The PPSU bulk is a hydrophobic environment, and so is the PPSU/air interface (air is hydrophobic). If air is more hydrophobic than the PPSU bulk environment, then the diffusion phenomenon is a possibility. The plasma treatment has a limited penetration depth, which is, however, deeper than the probe depth (5-10 nm) of the XPS analysis, which explains why no initial change is observed in the $\mathrm{C} / \mathrm{O} / \mathrm{S}$ composition, since it will take time for the plasma treated and non-plasma treated parts of the molecules to diffuse/rearrange. By extrapolating the temporal composition plot (Fig. 8) it becomes evident (not shown) that the composition will not reach the reference composition (within the expected lifetime of the membrane). It can thus be concluded that the plasma-induced surface properties will diminish in time but for all practical purposes not vanish. However, if the PPSU membrane is subsequently coated with a hydrophilic layer then the interactions between the hydrophilic oxidated PPSU and the hydrophilic layer could in principle prevent the diffusion phenomenon, i.e. arrest oxidation of PPSU. Unfortunately it is not straightforward to test this hypothesis as any subsequent coating prevents the buried PPSU surface from being analysed with surface sensitive techniques such as XPS.

\subsection{Separation properties of TFNC FO membrane}

To demonstrate the application of PPSU-based membrane support layers in TFC membrane fabrication we made and tested TFC-based biomimetic FO membranes. Specifically, aquaporin vesicles were incorporated into the active layer of the TFNC FO membrane as previously described. ${ }^{50}$ Aquaporinbased biomimetic membranes have drawn great attentions in the past decade, since aquaporin can provide ideally selective conduits for water with a high osmotic permeability. ${ }^{51,52}$

For the TFNC FO membrane prepared with PPSU ENM, $J_{\mathrm{W}}=$ $14.25 \mathrm{~L} \mathrm{~m}^{-2} \mathrm{~h}^{-1}$ and $J_{\mathrm{s}}=1.15 \mathrm{~g} \mathrm{~m}^{-2} \mathrm{~h}^{-1}$, corresponding to a specific reverse salt flux $J_{\mathrm{s}} / J_{\mathrm{w}}=0.08 \mathrm{~g} \mathrm{~L}^{-1}$. Membrane rejection for the fluorescent marker calcein $R_{\mathrm{Ca}}$ is $99.8 \%$. Compared to the commercially available aquaporin membrane all of the separation properties were improved, as listed in Table 2.

The membrane performance improvements of the PPSUsupported TFNC are attributed to two features of the electrospun membranes: a nanofibrous structure and high hydrophilicity. First, as shown in Fig. 9, the PPSU ENMs (Fig. 9(a)) provides much finer fibrous structure as compared to the commercial FO membrane support (Fig. 9(b)). In case of the electrospun PPSU membrane developed in this work, the heat treatments effectively promoted the formation of welding joints which transfer non-woven nanofiber structure into an interconnected 3-dimensional porous network. Such unique structures not only significantly enhance the permeability of water, but also provide a smooth interface with high surface area that can facilitate the interfacial polymerization during the formation of polyimide active layer. ${ }^{13}$ Second, unlike conventional polymer support layer materials (e.g. polysulfone), the PPSU ENMs after plasma treatments have
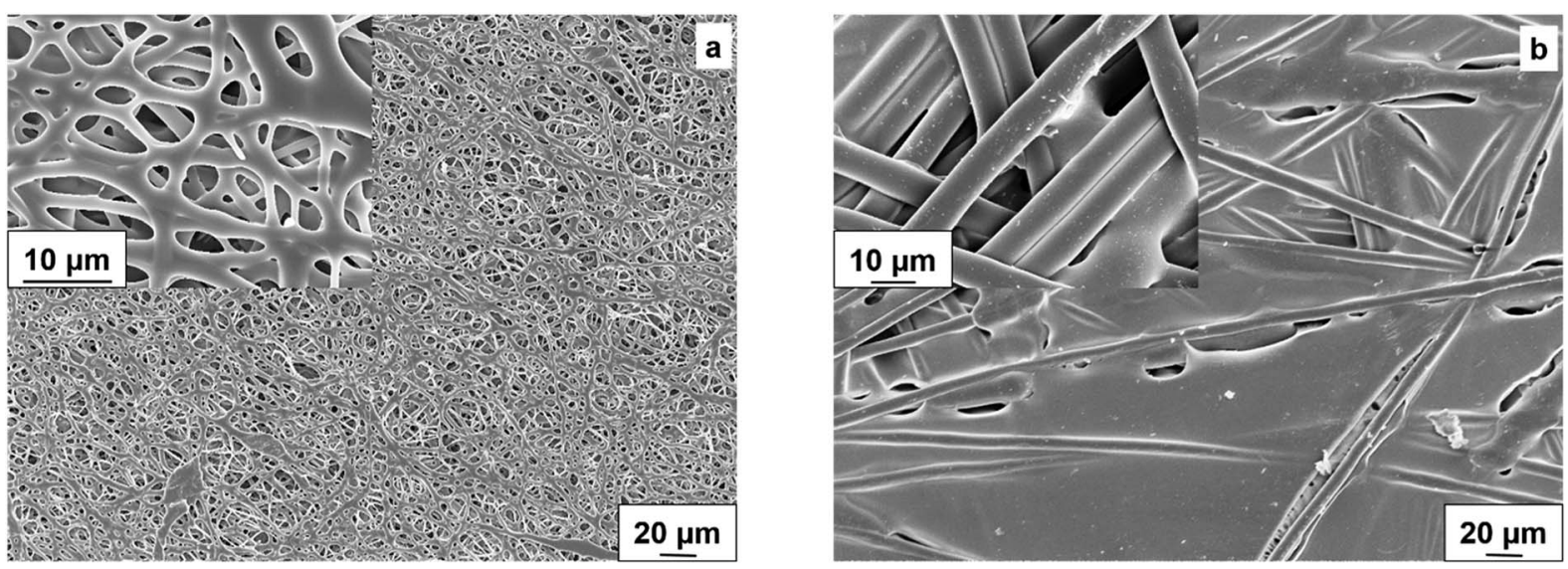

Fig. 9 SEM images of (a) PPSU ENMs and (b) commercial substrate membrane used in Aquaporin FO membranes. 
excellent hydrophilicity with contact angle of $0^{\circ}$. Such high hydrophilicity plays a crucial role in enhancing the water flux through the membrane in the osmotically driven membrane processes. $^{30}$

In addition, the PPSU membrane support layer has an elastic modulus 3.4 times larger than reported for modified PVDF nanofibers, ${ }^{31}$ which constitutes an important step towards reconciling the general demands for a thin, porous, yet strong membrane. FO processes have found their applications in fields varying from food and beverage industry, pharmaceutical industry to industrial and domestic wastewater treatment and power generation. ${ }^{53,54}$ Therefore, this TFNC FO membrane with PPSU ENM support layer can be applied in various industrial processes and has potential to improve efficiency and limit energy use and costs of these processes by means of improved water flux, reverse salt flux and rejection.

We foresee that further improvements in separation properties can be achieved by reducing the thickness of PPSU ENMs thereby reducing the resistance to mass transfer..$^{55}$ As the electrospinning technique allows precise tailoring of the thickness profile of prepared membranes - due to layer by layer deposition - it holds great potential in the design of FO-dedicated substrates. Although further research is needed to fully elucidate the correlation between the filtration properties and electrospun nanofiber structure, we conclude, based on the preliminary FO results presented, that PPSU ENM generally is a very promising material for developing support layers for TFCbased membranes.

The recyclability of the membrane materials used in water treatment should been taken into consideration, as the disposal of the end-of-life membrane modules has caused adverse environmental impacts. Several researches proposed to recycle TFC RO membranes by removing the polyamide active layer with oxidants. ${ }^{56,57}$ The results have shed light on the recyclability of the TFNC membrane in our study that after oxidation treatments the PPSU ENMs could be reused in less demanding processes, such as microfiltration or ultrafiltration.

\section{Conclusion}

In this present research, electrospinning combined with thermal treatment and plasma induced surface modification was utilized to develop an electrospun PPSU ENM as nanofiber support layers in biomimetic TFNC FO membranes. Thermal treatment effectively converted the fluffy, highly porous and mechanically weak nanofiber membrane into a more compact and tougher membrane with interfused 3-dimensional nanofiber network. These welding joints established during the heat treatment, significantly enhanced mechanical stability of the nanofiber membrane, with $930 \%$ increase in elastic modulus, $853 \%$ increase in maximum stress and nearly doubled the breaking strain. Low-pressure plasma treatment was proved to be an effective method for surface modification. With 2 min. plasma treatment on each side, there is a significant increase in oxygen content on the PPSU surface, resulting in a change of the contact angle from $137^{\circ}$ (non-plasma PPSU ENM) to $0^{\circ}$. The smooth and hydrophilic PPSU ENM provides an optimized surface for the coating of a polyamide active layer. As compared to the commercial membrane performance, the TFNC FO membrane shows significant enhancement in osmotic water flux and much lower reserve salt flux, owing to its highly interconnected pore structure and hydrophilic surface.

\section{Conflicts of interest}

There are no conflicts to declare.

\section{Acknowledgements}

The authors sincerely acknowledge H. C. Ørsted Postdoc Fellowship and Innovation Fund Denmark via the MEMENTO project. Dr Ing. Philippe Martin and Emanuele Di Nicolò at Solvay Specialty Polymers are sincerely acknowledged for providing PPSU polymers. Drs Krzysztof Trzaskus and Jörg Vogel, Aquaporin A/S, provided valuable support to the project.

\section{References}

1 T. Subbiah, G. S. Bhat, R. W. Tock, S. Parameswaran and S. S. Ramkumar, J. Appl. Polym. Sci., 2005, 96, 557-569.

2 S. Agarwal, A. Greiner and J. H. Wendorff, Prog. Polym. Sci., 2013, 38, 963-991.

3 H. Wu, W. Pan, D. Lin and H. Li, J. Adv. Ceram., 2012, 1, 2-23.

4 W. Zhang, M. W. Brodt and P. N. Pintauro, ECS Trans., 2011, 41, 891-899.

5 W. Zhang and P. N. Pintauro, ChemSusChem, 2011, 4, 17531757.

6 J. Choi, R. Wycisk, W. Zhang, P. N. Pintauro, K. M. Lee and P. T. Mather, ChemSusChem, 2010, 3, 1245-1248.

7 A. Enrico, W. Zhang, M. Lund Traulsen, E. M. Sala, P. Costamagna and P. Holtappels, J. Eur. Ceram. Soc., 2018, 38, 2677-2686.

8 S. B. Simonsen, J. Shao and W. Zhang, Nanotechnology, 2017, 28, 265402.

9 L. A. Mercante, V. P. Scagion, F. L. Migliorini, L. H. C. Mattoso and D. S. Correa, Trends Anal. Chem., 2017, 91, 91-103.

10 S. Thenmozhi, N. Dharmaraj, K. Kadirvelu and H. Y. Kim, J. Mater. Sci. Eng. B, 2017, 217, 36-48.

11 S. Thakkar and M. Misra, Eur. J. Pharm. Sci., 2017, 107, 148167.

12 G. Wang, D. Yu, A. D. Kelkar and L. Zhang, Prog. Polym. Sci., 2017, 75, 73-107.

13 X. Wang and B. S. Hsiao, Curr. Opin. Chem. Eng., 2016, 12, 62-81.

14 S. S. Ray, S.-S. Chen, C.-W. Li, N. C. Nguyen and H. T. Nguyen, $R S C A d v$., 2016, 6, 85495-85514.

15 Y. Liao, C.-H. Loh, M. Tian, R. Wang and A. G. Fane, Prog. Polym. Sci., 2018, 77, 69-94.

16 F. E. Ahmed, B. S. Lalia and R. Hashaikeh, Desalination, 2015, 356, 15-30.

17 K. Yoon, B. S. Hsiao and B. Chu, J. Membr. Sci., 2009, 326, 484-492. 
18 K. Yoon, K. Kim, X. Wang, D. Fang, B. S. Hsiao and B. Chu, Polymer, 2006, 47, 2434-2441.

19 X. Wang, D. Fang, K. Yoon, B. S. Hsiao and B. Chu, J. Membr. Sci., 2006, 278, 261-268.

20 H. Ma, C. Burger, B. S. Hsiao and B. Chu, Biomacromolecules, 2011, 12, 970-976.

21 T. Cath, A. Childress and M. Elimelech, J. Membr. Sci., 2006, 281, 70-87.

22 K. Lutchmiah, A. R. Verliefde, K. Roest, L. C. Rietveld and E. R. Cornelissen, Water Res., 2014, 58, 179-197.

23 X. Song, Z. Liu and D. D. Sun, Adv. Mater., 2011, 23, 32563260 .

24 N.-N. Bui, M. L. Lind, E. M. V. Hoek and J. R. McCutcheon, J. Membr. Sci., 2011, 385-386, 10-19.

25 N. N. Bui and J. R. McCutcheon, Environ. Sci. Technol., 2013, 47, 1761-1769.

26 L. Huang and J. R. McCutcheon, J. Membr. Sci., 2014, 457, 162-169.

27 M. Tian, C. Qiu, Y. Liao, S. Chou and R. Wang, Sep. Purif. Technol., 2013, 118, 727-736.

28 M. Tian, Y.-N. Wang and R. Wang, Desalination, 2015, 370, 79-86.

29 M. Tian, Y.-N. Wang, R. Wang and A. G. Fane, Desalination, 2017, 401, 142-150.

30 J. R. McCutcheon and E. Menachem, J. Membr. Sci., 2008, 318, 458-466.

31 L. Huang, J. T. Arena and J. R. McCutcheon, J. Membr. Sci., 2016, 499, 352-360.

32 M. Spluber and K. Trzaskus, WO 2017137361 A1, 2017.

33 A. Lacoste, T. Lagarde, S. B. chu, Y. Arnal and J. Pelletier, Plasma Sources Sci. Technol., 2002, 11, 407-412.

34 E. Stamate and M. Draghici, J. Appl. Phys., 2012, 111, 083303.

35 E. Stamate, Surf. Coat. Technol., 2014, 260, 401-410.

36 S. Kiani, S. M. Mousavi, N. Shahtahmassebi and E. Saljoughi, Desalin. Water Treat., 2015, 57, 16250-16259.

37 S.-S. Choi, Y. S. Lee, C. W. Joo, S. G. Lee, J. K. Park and K.-S. Han, Electrochim. Acta, 2004, 50, 339-343.

38 Y. Liang, S. Cheng, J. Zhao, C. Zhang, S. Sun, N. Zhou, Y. Qiu and X. Zhang, J. Power Sources, 2013, 240, 204-211.

39 L. Zhang, L.-G. Liu, F.-L. Pan, D.-F. Wang and Z.-J. Pan, J. Eng. Fibers Fabr., 2012, 7-16.
40 K. Tsougeni, N. Vourdas, A. Tserepi, E. Gogolides and C. Cardinaud, Langmuir, 2009, 25, 11748-11759.

41 D. S. Wavhal and E. R. Fisher, J. Membr. Sci., 2002, 209, 255269.

42 K. S. Kim, K. H. Lee, K. Cho and C. E. Park, J. Membr. Sci., 2002, 199, 135-145.

43 F. Yalcinkaya, B. Yalcinkaya, A. Pazourek, J. Mullerova, M. Stuchlik and J. Maryska, Int. J. Polym. Sci., 2016, 2016, 1-9.

44 S. Duan, X. Liu, Y. Wang, Y. Meng, A. Alsaedi, T. Hayat and J. Li, Plasma Processes Polym., 2017, 14, 1600218.

45 S. Duan, Y. Wang, X. Liu, D. Shao, T. Hayat, A. Alsaedi and J. Li, ACS Sustainable Chem. Eng., 2017, 5, 4073-4085.

46 S. Duan, X. Liu, Y. Wang, D. Shao, Y. Meng, T. Hayat, A. Alsaedi and J. Li, RSC Adv., 2017, 7, 21124-21127.

47 S. Duan, X. Xu, X. Liu, Y. Wang, T. Hayat, A. Alsaedi, Y. Meng and J. Li, J. Colloid Interface Sci., 2018, 513, 92-103.

48 H. Zhu, S. Duan, L. Chen, A. Alsaedi, T. Hayat and J. Li, Plasma Sci. Technol., 2017, 19, 115501.

49 K. Norrman, Y. Wang, E. Stamate and W. Zhang, Controlling surface properties of electrospun polyphenylsulfone using plasma treatment and X-ray photoelectron spectroscopy, Heliyon, under review.

50 Y. Zhao, C. Qiu, X. Li, A. Vararattanavech, W. Shen, J. Torres, C. Hélix-Nielsen, R. Wang, X. Hu, A. G. Fane and C. Y. Tang, J. Membr. Sci., 2012, 423-424, 422-428.

51 C. Tang, Z. Wang, I. Petrinić, A. G. Fane and C. Hélix-Nielsen, Desalination, 2015, 368, 89-105.

52 A. Giwa, S. W. Hasan, A. Yousuf, S. Chakraborty, D. J. Johnson and N. Hilal, Desalination, 2017, 420, 403-424.

53 A. Haupt and A. Lerch, Membranes, 2018, 38, 47.

54 S. Zhao, L. Zou, C. Y. Tang and D. Mulcahy, J. Membr. Sci., 2012, 396, 1-21.

55 K. L. Lee, R. W. Baker and H. K. Lonsdale, J. Membr. Sci., 1981, 8, 141-171.

56 E. Coutinho de Paula, J. C. L. Gomes and M. C. S. Amaral, Water Sci. Technol., 2017, 76, 605-622.

57 J. Landaburu-Aguirre, R. García-Pacheco, S. Molina, L. Rodríguez-Sáez, J. Rabadán and E. García-Calvo, Desalination, 2016, 393, 16-30. 